\title{
Adolescentes víctimas de bullying y ciberbullying en el Área Metropolitana de Monterrey (AMM)
}

\section{Teenage victims of bullying and cyberbullying in the Metropolitan Area of Monterrey, Mexico}

DOI: https://doi.org/10.32870/dse.v0i24.1025

\author{
Gloria Mancha Torres* \\ Arun Kumar Acharya**
}

\begin{abstract}
Resumen
Este trabajo analiza la incidencia del bullying y ciberbullying en los adolescentes de 12 a 16 años del AMM, empleando la Encuesta de Cohesión Social para la Prevención de la Violencia y la Delincuencia (ECOPRED, 2014). Nueve situaciones se clasificaron según tipo de bullying (físico, psicológico, social o sexual) y una situación como ciberbullying. Todas estas situaciones (excepto el acoso sexual y el cibernético) se presentan principalmente en la escuela y los compañeros son señalados como el principal agresor. El bullying tipo sexual se da principalmente en la propia casa de la víctima, siendo un desconocido o un integrante de su hogar el principal agresor. Por género, en el AMM, una mayor proporción de mujeres son víctimas de acoso de tipo físico y sexual, mientras una mayor proporción de hombres son víctimas de ciberbullying. Tanto en el AMM como en la media nacional, la incidencia de mujeres víctimas de bullying sexual es casi el doble que en hombres. La proporción de víctimas de ciberbullying en la media nacional es el doble que en el AMM, siendo las mujeres las principales víctimas.
\end{abstract}

Palabras clave: Bullying - ciberbullying - AMM - adolescentes - jóvenes.

\begin{abstract}
Using data from the Survey of Social Cohesion for the Prevention of Violence and Crime (ECOPRED) 2014, this paper analyzes the incidence of bullying and cyberbullying in young people aged 12 to 16 from the Metropolitan Area of Monterrey, Mexico (AMM). Nine situations were classified according to the type of bullying (physical, psychological, social, and sexual) and one situation was classified as cyberbullying. All these cyberbullying and bullying aggressions (except for the sexual harassment) took place mainly at school and schoolmates were identified as the main aggressors. In the case of sexual bullying, the aggression occurred at the victim's own house and either a stranger or a member of the household was identified as the aggressor. By gender, in the AMM, a greater proportion of women are victims of physical and sexual
\end{abstract}

\footnotetext{
* Doctora en Políticas Públicas. Líneas de investigación: Problemas de juventud (educación, empleo, violencia); género; grupos vulnerables; desarrollo sustentable. Facultad de Ciencias de la Comunicación, Universidad Autónoma de Nuevo León. México. glmancha@gmail.com

** Doctor en Antropología. Líneas de investigación: Migración; tráfico humano; género; estudios culturales. Sambalpur University, India / Instituto de Investigaciones Sociales (IINSO), UANL. acharya.iinso@gmail.com
} 
bullying, while a greater proportion of men are victims of cyberbullying. In both the AMM and the national average, the incidence of female victims of sexual bullying almost doubles that of males. The proportion of victims of cyberbullying in the national average doubles the incidence in the AMM, with women as the main victims.

Keywords: Bullying - cyberbullying - AMM - teenagers - youth people.

\section{Introducción}

En la última década, la violencia en México ha escalado a niveles que llevan a clasificar al país como uno de los más violentos en el ámbito mundial, ${ }^{1}$ y los jóvenes juegan un doble papel en el fenómeno al ocupar un lugar relevante tanto del lado de las víctimas como de los victimarios (Banco Mundial, 2012).

De acuerdo con diversos estudios, la violencia es un problema de salud pública grave, pues contribuye a aumentar sustancialmente las tasas de morbilidad y mortalidad, particularmente entre los jóvenes (Guerrero, 2008; Griffin et al, 1999; OMS, 2002); según las definiciones de la OMS y las estadísticas de violencia en México, el país enfrenta una epidemia de violencia, ${ }^{2}$ por lo que los jóvenes mexicanos viven en un clima de violencia que se generaliza y se observa tanto en la comunidad como en las escuelas y los hogares. Es decir, la violencia se ha convertido en un componente cotidiano de nuestras vidas, que está presente prácticamente en cualquier lugar donde se da una interacción entre individuos.

Según las teorías ambientales, la continua exposición a la violencia, en el hogar, el vecindario, la escuela o los medios de comunicación, promueve entre los niños y jóvenes la creencia de que la agresión y la violencia son conductas normales y aceptables, incrementando con ello el potencial de respuesta agresiva (López-Hernáez, 2015). En el mismo sentido, estudios empíricos han encontrado que niños y jóvenes expuestos a la violencia, ya sea como testigos o como víctimas, tienen un mayor riesgo de desarrollar patrones de comportamiento agresivo y violento (Fehon, 2007; Soto y Trucco, 2015).

La violencia es un fenómeno complejo y multidimensional que puede tener múltiples definiciones, por lo que con el objetivo de comparar datos, la OMS definió el término violencia como "el uso deliberado de la fuerza física o el poder, ya sea en grado de amenaza o efectivo, contra uno mismo, otra persona o un grupo o comunidad, que cause o tenga muchas probabilidades de causar lesiones, muerte, daños psicológicos, trastornos del desarrollo o privaciones" (OMS, 2002: 5). Así, dentro de esta definición se considera un abanico amplio de actos violentos que

1 México ocupa el lugar número 12 entre los países más violentos del mundo, con una tasa de 24.8 homicidios por cada 100 mil habitantes en 2017, según el Estudio Global de Homicidios 2019 del Centro de Información de la Organización de las Naciones Unidas (UNODC, 2019). 2 Según la OMS, una tasa de homicidios mayor a 10 por cada 100 mil habitantes es considerada una epidemia de violencia (OMS, 2002). 
van desde la intimidación y peleas hasta agresión grave y homicidios; entre estos actos catalogados como actos violentos, se encuentran el bullying o acoso escolar, y el ciberbullying. ${ }^{3}$

En el contexto de violencia en México, el buling se ubica como un problema social grave, incluso se le considera una "pandemia social", con base en su distribución geográfica en el país y el número de personas que afecta (Vega, 2013). En 2017 la OECD clasificó a México en el primer lugar en violencia verbal, física, psicológica y social entre los alumnos de educación básica. Por su parte el ciberbuling, es decir, el acoso a través de las tecnologías de la información y la comunicación (TIC) ha estado aumentando en forma considerable en todo el mundo y México no es la excepción (UNESCO, 2019; Domínguez et al., 2019).

Según la clasificación de entidades federativas de México, realizada por el Instituto Nacional de Evaluación Educativa (INEE, 2009), el promedio de violencia escolar en secundarias en 2008, los estados de Baja California Norte, Baja California Sur, Distrito Federal, Estado de México, Quintana Roo y Chihuahua se encontraban entre los que presentaban mayores niveles de violencia; mientras que Campeche, Puebla, Veracruz, Oaxaca y Chiapas estaban entre las entidades con los índices más bajos; Nuevo León se ubicó en la media nacional. La escala de violencia utilizada en dicho estudio se enfocó en conductas violentas consideradas casi como delictivas, más que en el acoso u hostigamiento entre alumnos (buling).

El objetivo de este trabajo es hacer un análisis de la incidencia del buling y ciberbuling en los jóvenes adolescentes de 12 a 16 años del Área Metropolitana de Monterrey (AMM), con base en datos de fuentes secundarias que nos permitan tener un diagnóstico general del fenómeno en esta área conurbada.

El trabajo está estructurado en seis secciones: la primera contiene un marco teórico conceptual del buling; la segunda, una revisión de literatura sobre trabajos realizados en México y el AMM; en la tercera sección se muestran los datos y la metodología que se siguió para este estudio; la cuarta contiene una descripción de los adolescentes en el AMM y en México, según datos de la misma ECOPRED; el análisis del buling y el ciberbuling en el AMM se presenta en la sección cinco; y finalmente, la sexta es una sección de conclusiones.

\section{Marco teórico-conceptual: El buling y su estudio}

Según González et al. (2018), se ha establecido un cierto tipo de consenso internacional para definir el término violencia, el cual incluye elementos clave como "ataques o intimidaciones físicas, verbales o psicológicas, que están destinadas a causar miedo, dolor o daño a la víctima"; en el mismo sentido, la definición de la OMS (2002) incluye elementos como el uso de la fuerza física o el poder, con el objetivo de causar lesiones, muerte, daño psicológico, etc. Así, el término violencia se utiliza para referirnos a una conducta que busca afectar de alguna forma a otro in-

3 A estos conceptos en adelante los denominaremos buling y ciberbuling como una manera de naturalizarlos en el idioma español [Nota de la correctora]. 
dividuo o a sí mismo, y esta afectación puede ser física o psicológica, y puede variar desde lesiones leves hasta causar la muerte. Dentro de este amplio espectro de situaciones se encuentran la violencia escolar, el buling y el ciberbuling.

Aunque el término violencia escolar es frecuentemente utilizado como sinónimo de buling, estos términos no son sinónimos ya que violencia escolar involucra un conjunto diverso de actos, actividades y acciones (como peleas, vandalismo y daños materiales, problemas de disciplina, disrupción en las aulas, etc.) que incluyen formas de agresión entre diferentes actores presentes en los centros escolares (alumnos, maestros, conserjes, directivos, etc.). Mientras que el concepto de buling está centrado en las agresiones o actos violentos entre pares o iguales cuando una de las partes se siente superior y ejerce algún tipo de violencia, agresión o maltrato sobre el otro; de esta forma, el buling es un tipo de violencia escolar (García, Ascensio, 2015; Acosta, 2014; Santoyo, Frías, 2014).

El estudio del fenómeno del buling se basa en teorías que nos permiten entender y comprender el comportamiento violento o agresivo de las personas, sobre todo de los niños y jóvenes. En cada teoría se consideran factores que pueden explicar el origen de la conducta violenta desde diferentes fundamentos, ya sea biológicos, emocionales, cognitivos, ambientales o sociológicos, e incluso, una mezcla de ellos (González et al., 2018; Acosta, 2014).

Según las teorías ambientales, el origen de la agresión se encuentra en el medio ambiente que rodea al agresor, en factores como la exposición repetida a la violencia en medios de comunicación, videojuegos, el clima de violencia en el hogar, la colonia o la ciudad en la que vive (López-Hernáez, 2015; Acosta, 2014).

Dentro del enfoque de las teorías ambientales, el modelo ecológico de Bronfenbrenner (1979) permite entender que la violencia es producto de la interrelación de factores que se encuentran en diversos niveles de influencia sobre el comportamiento del individuo, es decir, explora la relación entre los factores individuales (características de la persona, biológicas, cognitivas, motoras) y los factores que surgen del contexto o el entorno en el que la persona se desarrolla y se desenvuelve: las relaciones sociales con la familia, los amigos, la pareja; las relaciones que se dan en la comunidad, en la escuela, el lugar de trabajo, el vecindario; los patrones culturales, creencias e ideología (González, 2018; Santoyo, Frías, 2014).

Para las teorías del aprendizaje social, la agresión depende en buena medida de factores biológicos, es decir, que hay una predisposición biológica para participar en actos violentos, agresivos $u$ otras conductas desadaptadas, aunque se reconoce que el aprendizaje de la conducta se lleva a cabo dentro de la familia, en el grupo de iguales o en cualquier otro contexto en el que el individuo interactúa, por tanto, el aprendizaje puede bloquear o reforzar la predisposición biológica (González, 2018; López-Hernáez, 2015). Desde este enfoque, si ser agresivo o violento es una conducta que se aprende, no serlo también puede ser una conducta aprendida a través de modelos positivos de interacción. 
Entre estas teorías de aprendizaje social, el modelo de imitación social de Bandura (1973) y el modelo de contagio social de Berkowitz y Rawling (1963) suponen que el comportamiento agresivo es resultado del aprendizaje por medio de la imitación u observación de modelos cercanos afectivamente, ya sea de los padres, hermanos, amigos, etc. (López-Hernáez, 2015).

Finalmente, de acuerdo a la teoría sociológica, las situaciones sociales adversas, como la pobreza o marginación, son las principales causantes de la conducta violenta o agresiva a través de la frustración personal (Dollard et al., 1939). Aunque algunos autores observan que aquellos individuos que tienen un mayor equilibrio emocional pueden manejar de mejor manera la frustración y responder de una forma adecuada socialmente, es decir, sin agredir o violentar a otros (Berkowitz, 1969; López-Hernáez, 2015). Esto significa que mientras que hay factores que pueden detonar la violencia o la agresión, hay algunos otros que contribuyen a bloquear dicho comportamiento.

De acuerdo con las teorías presentadas, el comportamiento agresivo o violento depende entonces de múltiples factores, algunos de los cuales se consideran factores de protección ante dichas conductas (por ejemplo, las técnicas parentales adecuadas o un medio ambiente libre de violencia), mientras que otros se consideran factores de riesgo (por ejemplo, el inadecuado manejo de la frustración o los considerados biológicos). Así, un adecuado control de los factores de riesgo podría contribuir a disminuir los actos agresivos o violentos como el buling, el ciberbuling o la violencia escolar en niños y adolescentes.

A continuación, hacemos un abordaje conceptual del buling y el ciberbuling, así como los orígenes del estudio de estos fenómenos.

Los primeros estudios sobre buling se realizaron en la década de 1970 en los países escandinavos. Dan Olweus se considera el pionero en el tema, con la publicación de su libro Aggression in the Schools: Bullies and Whipping Boys, en 1978, donde describe el abuso de un grupo de alumnos sobre otros (Loredo et al., 2008; Aguilera et al., 2007; Castillo, Pacheco, 2008). A partir de entonces, tanto en los países europeos como en Estados Unidos y Japón, se inició el estudio de dicho fenómeno (García, Ascensio, 2015; Santoyo, Frías, 2014).

En México, los primeros estudios del tema se remontan a mediados de la década de 1990 (Prieto, 2005; Santoyo, Frías, 2014), y es en los últimos 20 años cuando la literatura científica en el tema se ha incrementado de manera significativa (Vega, González, 2016), sobre todo en los campos de la salud pública, la psicología, la sociología y la etnografía. Los estudios cuantitativos son en su mayor parte estudios de caso, pues los datos sobre el fenómeno en encuestas de representatividad nacional y estatal son muy limitados (Santoyo, Frías, 2014).

Buling es una palabra que deriva del vocablo sajón bully, que como sustantivo se podría traducir al español como "valentón" o "matón", y como verbo tendría el significado de maltratar o amedrentar (Loredo et al., 2008). Así, buling se utiliza para denominar el proceso de intimidación y victimización entre iguales, dentro de un aula o centro escolar (Aguilera et al., 2007; 
Ortega, Mora, 1997), en el que se ejerce un abuso sistemático y recurrente del poder. Para algunos investigadores, este proceso puede observarse, además de en el contexto escolar, en áreas laborales, recreativas, militares y, en general, en cualquier contexto donde los niños y adolescentes convivan, incluyendo la familia (Smith, 2000; García, Ascensio, 2015).

El buling se presenta con mayor frecuencia en la adolescencia temprana e intermedia ${ }^{4}$ y va disminuyendo conforme la edad aumenta (Loredo et al., 2008; Castillo, Pacheco, 2008); aunque en ocasiones tiende a considerarse como algo cotidiano $\mathrm{y}$, por lo tanto, normal o natural en esa etapa de la vida (Santoyo, Frías, 2014), se ha encontrado que en realidad deja huellas imborrables en la mayoría de las víctimas, e incluso en los victimarios. Por ejemplo, Anderson et al. (2001) analizaron varios estudios (realizados entre 1994 y 1999 en Estados Unidos) sobre muertes en las escuelas causadas por conductas violentas, y encontraron que los perpetradores de homicidios fueron individuos que habían sufrido violencia en el contexto escolar (buling). En el mismo sentido, otros estudios han encontrado que el buling se relaciona con diferentes formas de violencia juvenil, abuso de drogas, problemas emocionales, ansiedad y depresión, bajo desempeño académico e incluso con síntomas de enfermedad física e intentos de suicidio (Garaigordobil et al., 2018; Loredo et al., 2008; Cobo, Tello, 2008; Olweus, 1993). En el mismo sentido, la UNESCO (2019) alerta que el buling tiene un efecto negativo significativo en la salud mental, la calidad de vida y el rendimiento académico de niños y adolescentes, y que las víctimas de buling son más del doble de propensos a faltar a clases que aquellos que no lo sufren. López-Hernáez (2015) menciona que en resultados de diversos estudios se encontró que entre $70 \%$ y $90 \%$ de agresores adultos habían sido agresores cuando eran niños.

El buling se caracteriza por ser un comportamiento de abuso sistemático y recurrente en el tiempo, con la intención de infligir dolor físico y/o emocional, en el contexto de una relación desigual de poder entre agresor y víctima (Olweus, 1993). En este proceso se observan tres actores: i) el acosador o buly; ii) la víctima o el buleado; y, iii) los espectadores o testigos (González, Patlán, 2009; Loredo et al., 2008; Castillo, Pacheco, 2008); aunque de acuerdo con algunos autores se debería incluir un cuarto actor, que es aquel que tiene un doble rol: víctima/acosador, según la circunstancia (Mendoza et al., 2016).

En el caso del ciberbuling, la conducta violenta o de acoso se transfiere al espacio de las tecnologías de la información, principalmente internet (correo electrónico, mensajería instantánea o "chat", páginas web o blogs, redes sociales, etc.) y el teléfono móvil, ampliando con ello el alcance y la cantidad de espectadores, además de permitir un mayor anonimato, y por ende, impunidad para el agresor. La distancia física entre el agresor y su víctima, así como el anonimato que permite el ciberbuling, insensibiliza en mayor grado al agresor y le permite ejercer

4 No existe un consenso sobre las edades que corresponden a cada etapa de la adolescencia; según la definición de la OMS, la adolescencia temprana se presenta entre los 10 y los 13 años; la adolescencia media entre los 14 y los 16 años; y la adolescencia tardía entre los 17 y los 19 años (Mancha y Ayala, 2018). 
mayor violencia y crueldad hacia su víctima, mientras que a la víctima de esta situación le puede causar estrés emocional, malestar psicológico, ansiedad, depresión, trastornos del sueño y de alimentación, bajo rendimiento académico, una sensación de mayor vulnerabilidad e incluso ideas suicidas (Domínguez et al., 2019; Garaigordobil et al., 2018; Hernández, Solano, 2007).

Las formas de acoso o agresión a través de internet pueden ser muy variadas y de diferentes niveles de gravedad, e incluyen desde amenazas, calumnias, chantajes, discriminación, difamación, acoso psicológico, distribución de grabaciones de agresiones físicas o vejaciones, distribución de fotografías (algunas incluso editadas o modificadas), exclusión de grupos, entre otras (Domínguez et al., 2019; García, 2015).

Considerando la forma en que se lleva a cabo, el buling puede clasificarse en: i) Físico, cuando incluye conductas como golpes, empujones, patadas, quemaduras o cualquier otro daño corporal, encierro o daño a pertenencias; ii) Psicológico, cuando incluye amenazas e intimidación verbal y no verbal que provocan estados emocionales negativos en la víctima; iii) Social, cuando busca disminuir el prestigio de la víctima en el grupo o incluso su exclusión del mismo, e incluye burlas, insultos, apodos, esparcimiento de rumores, bromas insultantes, discriminación, entre otros; iv) Sexual, cuando involucra temas o conductas sexuales que ofenden o denigran a la víctima; $y$, v) Ciberbuling, que es un tipo de buling más reciente que surge a partir de los grandes avances tecnológicos y está asociado al uso del internet, los teléfonos celulares, computadoras, tabletas y redes sociales, e involucra uno o más de los tipos de buling anteriormente descritos (Garaigordobil et al., 2018; García, Ascensio, 2015; Valdés et al., 2012; Domínguez, Manzo, 2011).

Según el estudio Behind the Numbers: Ending School Violence and Bullying (UNESCO, 2019) que reúne datos de 144 países, el acoso físico es más común entre varones, mientras que el psicológico es más frecuente entre el sexo femenino; por otro lado, y de acuerdo al mismo estudio, el acoso sexual y el acoso en línea o por teléfono móvil han estado aumentando rápidamente.

\section{Revisión de literatura}

Como se mencionó anteriormente, en México los primeros estudios del buling se remontan a mitad de la década de 1990, y es en los últimos 20 años cuando la literatura científica sobre el tema se ha incrementado. En el caso del ciberbuling, los trabajos disponibles son aún muy escasos, a pesar de que México es uno de los países de Latinoamérica donde más se ha estudiado el tema, según Garaigordobil et al. (2018).

Algunos trabajos como el de Cobo y Tello (2008), Loredo et al. (2008) y García y Ascensio (2015), se enfocan a identificar y describir el fenómeno del buling. Otros trabajos aportan al estudio del tema en forma empírica a través de estudios de caso en una o varias escuelas, ya sea en investigaciones de corte cualitativo o cuantitativo.

Entre los estudios cualitativos se encuentran el de Prieto (2005), que busca entender cómo se genera la violencia escolar entre los jóvenes, a través de un estudio cualitativo en una 
secundaria pública en la Ciudad de México; el de Vázquez et al. (2005), quienes describen las percepciones de los jóvenes de una preparatoria de Guadalajara sobre la violencia; y el de Gómez (2005), quien realizó varios estudios de corte etnográfico en secundarias públicas de la Ciudad de México y contrastó estos datos con los recabados en otros estados del país.

Entre los estudios de caso de corte cuantitativo, se encuentran el de Domínguez y Manzo (2011), quienes realizaron una investigación cuantitativa exploratoria con una muestra de tres secundarias de Morelia, Michoacán; el de Vega y González (2016), donde se elige una muestra representativa de las secundarias públicas del área metropolitana de Guadalajara, Jalisco, con el objetivo de identificar la prevalencia de adolescentes agresores, así como analizar los factores que disuaden o alientan el comportamiento agresivo; el de Valdés et al. (2012), quienes realizaron un estudio cuantitativo con una muestra de diez secundarias públicas del estado de Sonora, con el objetivo de identificar las variables familiares que diferencian a los estudiantes que reportan conductas violentas de aquellos que no presentan esta conducta; el de Castillo y Pacheco (2008), que tiene el objetivo de evaluar la incidencia entre iguales en estudiantes de secundaria, a través de una encuesta a 280 estudiantes en total, de una muestra de 18 secundarias de Mérida, Yucatán; y el de González et al. (2018), quienes investigan los tipos de violencia y los factores familiares que originan los comportamientos violentos de adolescentes en preparatorias técnicas de Nuevo León, a través del análisis de 210 entrevistas a alumnas de tres preparatorias en el área metropolitana de Monterrey.

Por otra parte, los estudios de ciberbuling en México son muy escasos, entre ellos se encuentran el de Del Río et al. (2009), quienes estudiaron el acoso digital en escolares de 10 a 18 años en varios países de Latinoamérica, y entre sus resultados mencionan que en México $14.7 \%$ de los estudiantes en este rango de edad son víctimas de ciberacoso; el de Lucio (2009), quien hizo un estudio entre jóvenes de preparatoria de la UANL para evaluar la prevalencia del ciberbuling entre estudiantes de esta universidad, y encontró que $20 \%$ de estos jóvenes eran ciberagresores y que las agresiones incluían insultos, amenazas, acoso sexual, chantajes, mensajes desagradables, comentarios difamatorios, rumores con el objetivo de desprestigiar, envío de videos desagradables, fotografías subidas para desprestigiar o ridiculizar, robo de contraseñas y hackeo de cuentas; por su parte, García-Maldonado et al. (2012) realizaron un estudio entre adolescentes de 11 a 15 años (estudiantes de una secundaria en Tampico, Tamaulipas) con el objetivo de determinar la prevalencia del ciberbuling, y encontraron que $3.5 \%$ de estos jóvenes eran víctimas, $2.8 \%$ eran agresores y $1.3 \%$ tenían el doble rol de víctimas/agresores; mientras que Vega-López et al. (2013), llevaron a cabo un estudio entre jóvenes de 12 a 15 años de secundarias públicas en Tlaquepaque, Jalisco, para identificar la prevalencia de víctimas de ciberacoso y las características de la agresión que llevaban a cabo, y encontraron que 14.3\% de estos jóvenes eran víctimas de ciberbuling y que las principales formas de agresión eran la trasmisión de textos e imágenes insultantes y los mensajes de texto e imágenes negativas; además, encontraron una mayor prevalencia entre el sexo masculino. 
Así, de los artículos sobre el tema realizados en México, resaltan en cantidad los estudios de caso muy específicos de una escuela o grupo de escuelas, lo cual es muy valioso para la aplicación de políticas concretas y focalizadas; sin embargo, y considerando lo que menciona Ortega (2010) acerca de que el problema de la violencia escolar no debe generalizarse desde un enfoque de política pública basada en evidencia, sino que es necesario observar el panorama y tener datos de lo que pasa en una ciudad o estado, hacer una comparación con otras ciudades y verificar, a través del tiempo, si el problema analizado mejora o no.

Dado que los estudios de estos fenómenos para Nuevo León y el AMM son muy escasos, el objetivo principal de este trabajo es hacer un análisis de la incidencia del buling y ciberbuling en los adolescentes de 12 a 16 años, que nos permita tener un panorama de cómo se encuentra esta área metropolitana respecto a la media nacional y conocer cuáles son las situaciones de buling más frecuentes. Esta información podría ser evidencia para la elaboración de una política pública focalizada en el AMM sobre el tema.

\section{Datos y metodología}

Este estudio de corte cuantitativo para el Área Metropolitana de Monterrey está basado en los datos de la Encuesta de Cohesión Social para la Prevención de la Violencia y la Delincuencia (ECOPRED, 2014). Esta encuesta es la primera de su tipo que se realiza en México y tiene el objetivo de medir factores de riesgo y exposición a situaciones de violencia y delincuencia de jóvenes de 12 a 29 años en 47 ciudades o áreas metropolitanas mexicanas; es representativa para estas zonas urbanas, pero no para los estados. La información proviene de dos cuestionarios, uno aplicado al jefe del hogar y uno aplicado al joven. Los datos que arroja la ECOPRED son muy valiosos para realizar estudios de corte transversal acotados al año en que fue realizada la encuesta; sería un buen instrumento para hacer estudios longitudinales, sin embargo, no se ha llevado a cabo, ni está planeado aún, que esta encuesta se realice de forma periódica.

Este trabajo se enfoca en jóvenes de 12 a 16 años 5 ya que, como se encuentra en la literatura del tema, el fenómeno se presenta con mayor frecuencia en la adolescencia temprana y media (Loredo et al., 2008; Castillo, Pacheco, 2008), esto sería entre los 10 y 16 años (según la definición etaria de adolescencia de la OMS); sin embargo, esta encuesta presenta información de los jóvenes a partir de los 12 años.

Con base en las definiciones de buling y ciberbuling y sus diferentes tipos que aparecen en la literatura presentada anteriormente, se construyeron variables proxy a partir de las preguntas del cuestionario del módulo para jóvenes de la ECOPRED. Se detectaron nueve situaciones que pueden ser definidas como buling y una como ciberbuling. Las nueve situaciones fueron clasificadas dentro de alguna categoría o tipo específico de buling (físico, psicológico, social o sexual); así, dentro de la categoría de buling físico se consideraron cuatro situaciones: i) si le han

5 En adelante, cuando hablamos de adolescentes, nos referimos a la población en este rango de edad. 
hecho algún daño corporal o lastimado su cuerpo; ii) si le han dañado o escondido sus objetos personales; iii) si le han robado alguna pertenencia sin que se haya dado cuenta; $y$ iv) si le han robado con violencia. En la categoría de buling psicológico se consideraron dos situaciones: i) si alguien lo ha amenazado o intimidado; y, ii) si alguien le ha exigido algo para no molestarlo, es decir, lo ha extorsionado. En buling social, únicamente se consideró una situación: i) si alguien se ha burlado, le ha puesto apodos hirientes o ha esparcido rumores o mentiras de su persona o si lo ha excluido por sus gustos, físico o ropa. En la categoría de buling sexual se consideraron dos situaciones: i) si alguien ha tocado o intentado tocar su cuerpo; $y$ ii) si alguien lo ha obligado o intentado obligar a ver o realizar alguna práctica sexual. Por su parte, se clasificó como ciberbuling la situación en la que alguien ha distribuido un mensaje de texto, imagen o video de su persona sin su consentimiento, para chantajear, humillar o acosar.

En los siguientes apartados, a partir de los datos de la ECOPRED, se presentan las características generales de los adolescentes de 12 a 16 años tanto en el AMM como en la media nacional y, posteriormente, se presenta el análisis del buling y ciberbuling específicamente para los jóvenes adolescentes del AMM.

\section{Los adolescentes en el AMM y en México}

En 2014, la población de Nuevo León era de 5.06 millones de personas y alrededor de 80\% de esta población se concentraba en los municipios del AMM. Los jóvenes de 12 a 16 años, por su parte, representaban $8.97 \%$ de la población del AMM, es decir, había 364,700 jóvenes adolescentes en este rango de edad, de los cuales $48.8 \%$ eran mujeres y $51.2 \%$ eran hombres.

En México, había 11.54 millones de adolescentes en 2014, de los cuales $49.3 \%$ eran mujeres y $50.7 \%$ eran hombres. Así, en el AMM habitaba 3.16\% de los adolescentes de 12 a 16 años del país.

Según datos de la ECOPRED 2014, los adolescentes del AMM viven en hogares con ligeramente menos habitantes que los adolescentes de la media nacional ${ }^{6}$ de México, así, un hogar promedio con adolescentes en el AMM tiene 4.98 integrantes, mientras que el hogar promedio de un adolescente en el mismo rango de edad en México tiene 5.07 miembros. $87.5 \%$ de los adolescentes del AMM viven en una vivienda propia, mientras en la media nacional esta proporción se ubica en $72.8 \%$.

Por tipo de actividad, se observa que $88.8 \%$ de los adolescentes en el AMM estudiaba, mientras en la media nacional esta proporción se ubicaba en $89.1 \%$; de los adolescentes del AMM $3.8 \%$ trabajaba y, por su parte, en la media nacional esta proporción era 3.4\%; el desempleo en el AMM era $0.7 \%$, mientras que en la media nacional era $0.5 \% ; 2 \%$ de los adolescentes de México y $1.8 \%$ de los del AMM se dedicaban al hogar; finalmente, el porcentaje de adolescentes que de-

6 Por cuestiones de espacio y simplificación de términos, con media nacional nos referimos a la media de las 47 ciudades para las que los datos de la ECOPRED son representativos. 
claraban no hacer nada, es decir, que podrían ser clasificados como “Ninis", era 4.6\% en el AMM y $4.7 \%$ en la media nacional en 2014 (tabla 1).

Tabla 1. Adolescentes de 12 a 16 años por tipo de actividad, AMM y México, 2014

\begin{tabular}{|l|c|c|}
\hline & AMM & México \\
\hline Estudia & 88.8 & 89.1 \\
\hline Trabaja & 3.8 & 3.4 \\
\hline Está desempleado & 0.7 & 0.5 \\
\hline Se dedica al hogar & 1.8 & 2.0 \\
\hline No hace nada & 4.6 & 4.7 \\
\hline
\end{tabular}

Fuente: ECOPRED (INEGI, 2015).

\section{El buling y ciberbuling en el AMM}

En México en 2014, 47.3\% de los adolescentes declararon haber sufrido alguna situación que puede ser clasificada como buling o ciberbuling, por lo menos una vez durante el último año, mientras que en el AMM esta cifra se ubicó en $37.1 \%$, esto es, 10 puntos porcentuales por debajo de la media nacional (tabla 2).

Tabla 2. Adolescentes de 12 a 16 años por tipo de buling (\%) AMM y México, 2014

\begin{tabular}{|l|c|c|}
\hline Tipo de Buling & AMM & México \\
\hline Físico & $\mathbf{2 7 . 9}$ & 37.1 \\
\hline Daño corporal & 7.6 & 10.0 \\
\hline Daño a objetos & 14.9 & 18.0 \\
\hline Robo & 14.5 & 22.9 \\
\hline Robo con violencia & 3.7 & 5.0 \\
\hline Psicológico & $\mathbf{4 . 2}$ & 7.5 \\
\hline Amenaza & 3.9 & 6.1 \\
\hline Extorsión & 0.3 & 2.2 \\
\hline Social & $\mathbf{2 1 . 6}$ & $\mathbf{2 5 . 1}$ \\
\hline Burla & 21.6 & 25.1 \\
\hline Sexual & 3.2 & $\mathbf{5 . 5}$ \\
\hline Tocamiento & 2.4 & 4.8 \\
\hline Situación sexual indeseada & 1.1 & 1.5 \\
\hline Ciberbuling & $\mathbf{1 . 2}$ & $\mathbf{2 . 5}$ \\
\hline Cualquiera de las anteriores & 37.1 & 47.3 \\
\hline
\end{tabular}

Fuente: ECOPRED (INEGI, 2015). 
Si se analiza cada uno de los tipos de buling y las situaciones específicas que pueden ser clasificadas como tal, se observa que en todos los casos las cifras para el AMM se ubican por debajo de la media nacional y que tanto en el AMM como en la media nacional el buling de tipo físico es el que los adolescentes más sufren; en el AMM 27.9\% de los adolescentes enfrentó por lo menos una de las cuatro situaciones que se pueden clasificar como buling físico en el año, mientras que en la media nacional, $37.1 \%$ de los adolescentes le ha pasado por lo menos una de estas situaciones.

En el AMM, 14.9\% de los adolescentes declaró haber sufrido daño a sus objetos personales con la intención de molestarlo y $14.5 \%$ mencionó que le han robado alguna pertenencia sin que se diera cuenta; $7.6 \%$ declaró que alguien había lastimado su cuerpo con malas intenciones, ya sea mediante jalones de cabello, empujones, pellizcos, golpes, entre otros, provocándole dolor físico, moretones, cortadas, quemaduras, fracturas, etc.; y 3.7\% declaró que por lo menos una vez le habían arrebatado sus cosas con violencia u obligado a entregar alguna pertenencia con algún tipo de agresión física o verbal. En la media nacional, el robo se ubicó como la situación de buling físico con mayor incidencia (22.9\%), seguida del daño a objetos (18\%), daño corporal (10\%) y robo con violencia (5\%).

Entre los adolescentes del AMM, 4.2\% sufrió algún tipo de situación que puede ser clasificada como buling psicológico, ya sea amenazas de lastimar físicamente a su persona, un familiar o un amigo (3.9\%), o una extorsión (0.3\%), es decir, se le ha exigido dinero, objetos o favores para no molestarlo o dejarlo hacer sus actividades. En la media nacional, a 7.5\% de los adolescentes les pasó una situación clasificada como buling psicológico, ya sea amenazas (6.1\%) o extorsión (2.2\%).

En cuanto al buling de tipo social, es decir, burlas, apodos hirientes, rumores o mentiras y la exclusión por los gustos, el físico o la ropa, en el AMM se ubicó en 21.6\%, mientras en la media nacional fue $25.1 \%$. Si se consideran las cifras por situación específica, esta situación es la que presenta las cifras más altas, tanto en el AMM como en la media nacional.

Respecto a las situaciones sexuales, se obtuvo información para dos tipos que pueden ser clasificadas como buling de tipo sexual; en el AMM, 3.2\% de los adolescentes mencionó que por lo menos una vez le pasó una de las dos situaciones siguientes: alguien tocó o intentó tocar alguna parte de su cuerpo y lo hizo sentir mal (2.4\%) y/o, alguien lo obligó o intentó obligarlo a ver o realizar alguna práctica sexual (1.1\%). En la media nacional, 5.5\% de los adolescentes enfrentó alguna de las situaciones de buling de tipo sexual, específicamente, 4.8\% mencionaron la situación de tocamiento y $1.5 \%$ una situación sexual indeseada.

Por último, en cuanto al ciberbuling, 1.2\% de los adolescentes del AMM mencionaron haber sido víctimas de alguien que ha distribuido un mensaje de texto, imagen o video de su persona sin su consentimiento para chantajear, acosar o humillar. En la media nacional, esta proporción es el doble que en el AMM, pues se ubica en 2.5\%. Estas cifras son para 2014 y según, la UNESCO 
(2019), el ciberbuling ha estado aumentando rápidamente en los últimos años, por lo que ante la falta de datos más recientes sobre este fenómeno para el AMM, sería necesario buscar la forma de generarlos.

Como puede observarse en la tabla 3, en un análisis por género, en el AMM $37.4 \%$ de las mujeres fueron víctimas de buling, mientras que la proporción de hombres se ubicó en 36.7\%; por su parte, en la media nacional, tanto hombres como mujeres fueron víctimas de buling en la misma proporción (47.3\%).

Tabla 3. Adolescentes de 12 a 16 años por tipo de buling según género (\%) AMM y México, 2014

\begin{tabular}{|l|c|c|c|c|}
\hline & \multicolumn{2}{|c|}{ AMM } & \multicolumn{2}{c|}{ México } \\
\hline Buling & Mujeres & Hombres & Mujeres & Hombres \\
\hline Físico & 30.6 & $\mathbf{2 5 . 2}$ & 36.0 & 38.1 \\
\hline Daño corporal & 9.3 & 6.0 & 10.2 & 9.9 \\
\hline Daño a objetos & 15.9 & 13.9 & 16.0 & 20.0 \\
\hline Robo & 16.9 & 12.2 & 23.7 & 22.0 \\
\hline Robo con violencia & 3.9 & 3.5 & 3.6 & 6.4 \\
\hline Psicológico & $\mathbf{4 . 2}$ & $\mathbf{4 . 2}$ & $\mathbf{7 . 0}$ & $\mathbf{8 . 0}$ \\
\hline Amenaza & 4.2 & 3.6 & 5.7 & 6.4 \\
\hline Extorsión & 0.0 & 0.6 & $\mathbf{2 . 0}$ & 2.3 \\
\hline Social & $\mathbf{2 1 . 4}$ & $\mathbf{2 1 . 7}$ & $\mathbf{2 6 . 4}$ & $\mathbf{2 3 . 8}$ \\
\hline Burla & 21.4 & 21.7 & 26.4 & 23.8 \\
\hline Sexual & $\mathbf{4 . 2}$ & $\mathbf{2 . 2}$ & $\mathbf{7 . 6}$ & $\mathbf{3 . 4}$ \\
\hline Tocamiento & 3.6 & 1.2 & 6.9 & $\mathbf{2 . 7}$ \\
\hline $\begin{array}{l}\text { Situación sexual } \\
\text { indeseada }\end{array}$ & $\mathbf{1 . 1}$ & 1.0 & 1.9 & 1.1 \\
\hline Ciberbuling & $\mathbf{0 . 6}$ & $\mathbf{1 . 8}$ & $\mathbf{3 . 0}$ & $\mathbf{1 . 9}$ \\
\hline $\begin{array}{l}\text { Cualquiera de las } \\
\text { anteriores }\end{array}$ & 37.4 & 36.7 & $\mathbf{4 7 . 3}$ & $\mathbf{4 7 . 3}$ \\
\hline
\end{tabular}

Fuente: ECOPRED (INEGI, 2015).

Por tipo de buling, en el AMM una mayor proporción de mujeres son víctimas del tipo físico y sexual, mientras una mayor proporción de hombres son víctimas de ciberbuling; la misma proporción de mujeres que hombres son víctimas de buling psicológico y de social. Por su parte, en la media nacional hay una mayor proporción de hombres víctimas de buling físico y psicológico, mientras una mayor proporción de mujeres son víctimas de buling social, sexual y ciberbuling.

Tanto en el AMM como en la media nacional, las mujeres son más frecuentemente víctimas de buling sexual que los hombres. En específico, en la situación de tocamiento o intento de tocamiento, la proporción de mujeres que son víctimas es más del doble que la de hombres, 
tanto en el AMM como en la media nacional.

En el AMM una mayor proporción de mujeres son víctimas de todas las situaciones de buling físico, de todas las situaciones de buling sexual y de amenazas (tipo psicológico). Por su parte, una mayor proporción de hombres son víctimas de extorsión (tipo psicológico) y de ciberbuling.

Otra parte importante del análisis es conocer el lugar donde cada situación se presentó (tabla 4) y quién fue el agresor o buly (tabla 5) pues con esta información es posible generar política pública con evidencia, y focalizada para proteger a los adolescentes de situaciones de acoso.

Tabla 4. Adolescentes de 12 a 16 años por tipo de buling según lugar donde pasó la situación (\%) AMM 2014

\begin{tabular}{|l|c|c|c|c|c|c|}
\hline Buling & Casa & Escuela & Trabajo & Lugar público* & Calle & Otro \\
\hline Físico & $\mathbf{1 1 . 2}$ & $\mathbf{7 5 . 5}$ & $\mathbf{4 . 0}$ & $\mathbf{6 . 6}$ & $\mathbf{1 7 . 9}$ & $\mathbf{4 . 2}$ \\
\hline Daño corporal & 14.5 & 56.7 & 3.6 & 2.7 & 22.0 & 4.0 \\
\hline Daño a objetos & 9.6 & 83.5 & 3.8 & 1.7 & 8.7 & 2.1 \\
\hline Robo & 5.9 & 73.1 & 1.9 & 7.5 & 15.0 & 1.9 \\
\hline Robo con violencia & 13.9 & 44.8 & 0.0 & 7.9 & 31.7 & 7.7 \\
\hline Psicológico & $\mathbf{0 . 0}$ & $\mathbf{6 2 . 1}$ & $\mathbf{0 . 0}$ & $\mathbf{1 3 . 6}$ & $\mathbf{3 8 . 1}$ & $\mathbf{0 . 0}$ \\
\hline Amenaza & 0.0 & 59.3 & 0.0 & 14.6 & 33.6 & 0.0 \\
\hline Extorsión & 0.0 & 100.0 & 0.0 & 0.0 & 100.0 & 0.0 \\
\hline Social & $\mathbf{5 . 7}$ & $\mathbf{6 8 . 0}$ & $\mathbf{1 . 1}$ & $\mathbf{1 0 . 5}$ & $\mathbf{2 4 . 4}$ & $\mathbf{2 . 6}$ \\
\hline Burla & 5.7 & 68.0 & 1.1 & 10.5 & 24.4 & 2.6 \\
\hline Sexual & $\mathbf{1 7 . 5}$ & $\mathbf{7 . 9}$ & $\mathbf{0 . 0}$ & $\mathbf{7 . 9}$ & $\mathbf{8 . 2}$ & $\mathbf{1 4 . 1}$ \\
\hline Tocamiento & 23.2 & 10.5 & 0.0 & 10.5 & 10.8 & 18.7 \\
\hline Situación sexual indeseada & 0.0 & 0.0 & 0.0 & 0.0 & 0.0 & 0.0 \\
\hline Ciberbuling & $\mathbf{0 . 0}$ & $\mathbf{4 9 . 5}$ & $\mathbf{0 . 0}$ & $\mathbf{0 . 0}$ & $\mathbf{0 . 0}$ & $\mathbf{5 0 . 5}$ \\
\hline Cualquiera de las anteriores & 11.8 & 72.1 & 3.7 & 11.1 & 30.4 & 6.7 \\
\hline *Plaza, mercado, parque, etc. & & & & & & \\
\hline
\end{tabular}

Fuente: ECOPRED (INEGI, 2015).

En el AMM, 72.1\% de los adolescentes que sufrieron por lo menos una de las situaciones de buling o ciberbuling mencionaron que el lugar donde se presentó esta situación fue en la escuela; $30.4 \%$ mencionaron haberlo sufrido en la calle; $11.8 \%$ que fueron víctimas en su casa; $11.1 \%$ que alguna de las situaciones que enfrentaron se dio en un lugar público, por ejemplo, 
en una plaza, mercado o parque; $6.7 \%$ mencionó que fue en otro lugar y $3.7 \%$ en su trabajo. ${ }^{7}$

De esta forma, la escuela es el principal lugar donde los adolescentes del AMM son víctimas de buling o ciberbuling, siguiendo en importancia, aunque por más de 40 puntos porcentuales por debajo, la calle. Este resultado está en sintonía con los hallazgos que se encuentran en la literatura, donde se considera que la escuela es el lugar donde más se presenta el buling, por lo que el término se utiliza como sinónimo de acoso escolar.

En el AMM, el buling físico se presenta principalmente en la escuela (75.5\%), la calle (17.9\%) y la casa (11.2\%). Respecto a las situaciones específicas, los adolescentes mencionaron que los episodios donde alguien ha dañado o lastimado su cuerpo se presentaron en la escuela (56.7\%), en la calle (22\%) y en su casa (14.5\%). La situación en la que alguien dañó o escondió sus objetos se presentó principalmente en la escuela (83.5\%), en su casa (9.6\%) y la calle (8.7\%). El robo sin violencia se presentó en la escuela (73.1\%), en la calle (15\%) y en algún lugar público (7.5\%). Por su parte, el robo con violencia se presentó en la escuela (44.8\%), en la calle (31.7\%) y en su casa (13.9\%).

El buling psicológico hacia los adolescentes en el AMM se presentó en la escuela (62.1\%), en la calle (38.1\%) y en un lugar público (13.6\%). En cuanto a las situaciones específicas, se observa que las extorsiones se cometen en la escuela y en la calle (la extorsión es el tipo de buling que tiene la menor incidencia en el AMM, con únicamente $0.3 \%)$; mientras que las amenazas suceden principalmente en la escuela (59.3\%), en la calle (33.6\%) y en un lugar público (14.6\%).

El buling de tipo social, es decir, las burlas, apodos, exclusión, etc., se presentó principalmente en la escuela (68\%), la calle (24.4\%) y los lugares públicos (10.5\%). Por otra parte, los adolescentes del AMM mencionaron que fueron víctimas de ciberbuling en la escuela (49.5\%) y en otro lugar (50.5\%).

Finalmente, en cuanto al buling de tipo sexual, se observa que los adolescentes mencionaron ser víctimas de esta situación principalmente en su casa (17.5\%), en otro lugar (14.1\%), en la calle (8.2\%), en la escuela (7.9\%) y en un lugar público (7.9\%). Cabe mencionar que este tipo de buling es el único donde la escuela no es el principal lugar de incidencia. Por tipo de situación específica, se observa que los adolescentes no mencionaron el lugar donde alguien lo obligó o intentó obligarlo a ver o realizar alguna práctica sexual, sin embargo, como se verá más adelante, sí mencionan quién fue el agresor. En cuanto a la situación donde alguien lo tocó o intentó tocarlo, los adolescentes mencionaron que este tipo de situación se presentó principalmente en su casa (23.2\%), en otro lugar (18.7\%), en la calle (10.8\%) y en la escuela o un lugar público $(10.5 \%)$, respectivamente.

7 La suma de los porcentajes no es 100\%, porque algunos adolescentes mencionan que la o las situaciones en las que han sido víctimas se han dado en varios lugares. 
En resumen, se observa que los adolescentes del AMM son víctimas de buling físico, psicológico, social y ciberbuling principalmente en la escuela y que el de tipo sexual sucede principalmente en la propia casa del adolescente.

Una vez que se conoce la información de dónde se cometen los actos de buling contra los adolescentes en el AMM, se analiza quién es la persona que comete estos actos, es decir, quién es el agresor o buly (tabla 5).

Tabla 5. Adolescentes de 12 a 16 años por tipo de buling según quién fue el agresor (buly) (\%) AMM 2014

\begin{tabular}{|l|c|c|c|c|c|c|c|}
\hline Buling Agresor & $\begin{array}{c}\text { Integrante } \\
\text { de su hogar }\end{array}$ & Su pareja & $\begin{array}{c}\text { Compañero } \\
\text { de escuela }\end{array}$ & $\begin{array}{c}\text { Compañero } \\
\text { de trabajo }\end{array}$ & $\begin{array}{c}\text { Familiar, } \\
\text { amigo o } \\
\text { conocido }\end{array}$ & $\begin{array}{c}\text { Conocido } \\
\text { de vista }\end{array}$ & $\begin{array}{c}\text { Desco- } \\
\text { nocido }\end{array}$ \\
\hline Físico & 5.9 & 0.9 & 68.1 & 4.1 & 13.5 & 10.5 & 18.2 \\
\hline Daño corporal & 14.5 & 0.0 & 56.5 & 3.6 & 15.2 & 5.9 & 7.9 \\
\hline Daño a objetos & 1.6 & 1.7 & 75.9 & 3.8 & 9.4 & 3.6 & 7.3 \\
\hline Robo & 2.0 & 0.0 & 64.9 & 2.0 & 4.0 & 11.4 & 19.2 \\
\hline Robo con violencia & 6.0 & 0.0 & 28.2 & 0.0 & 31.6 & 15.8 & 24.4 \\
\hline Psicológico & 0.0 & 0.0 & 61.3 & 0.0 & 7.3 & 18.3 & 13.1 \\
\hline Amenaza & 0.0 & 0.0 & 58.5 & 0.0 & 7.8 & 19.6 & 14.0 \\
\hline Extorsión & 0.0 & 0.0 & 100.0 & 0.0 & 0.0 & 0.0 & 0.0 \\
\hline Social & 2.9 & 0.0 & 68.5 & 1.3 & 14.5 & 9.3 & 13.0 \\
\hline Burla & 2.9 & 0.0 & 68.5 & 1.3 & 14.5 & 9.3 & 13.0 \\
\hline Sexual & 17.3 & 0.0 & 0.0 & 0.0 & 16.3 & 9.7 & 31.5 \\
\hline Tocamiento & 12.1 & 0.0 & 0.0 & 0.0 & 21.6 & 0.0 & 41.7 \\
\hline $\begin{array}{l}\text { Situación sexual } \\
\text { indeseada }\end{array}$ & 24.9 & 0.0 & 0.0 & 0.0 & 0.0 & 29.7 & 0.0 \\
\hline Ciberbuling & 0.0 & 0.0 & 76.4 & 0.0 & 0.0 & 0.0 & 23.6 \\
\hline $\begin{array}{l}\text { Cualquiera de las } \\
\text { anteriores }\end{array}$ & 6.8 & 0.7 & 67.6 & 3.8 & 16.2 & 15.7 & 22.9 \\
\hline
\end{tabular}

Fuente: ECOPRED (INEGI, 2015).

Como se puede observar en la tabla anterior, $67.6 \%$ de los adolescentes del AMM mencionaron que su agresor fue un compañero de la escuela; $22.9 \%$, que su agresor era un desconocido; $16.2 \%$ reportó a un familiar, amigo o conocido; $15.7 \%$ indicó que fue víctima de un conocido de vista; $6.8 \%$, que el agresor es un integrante de su hogar; $3.8 \%$ señaló como agresor a un compañero de trabajo, y $0.7 \%$ dijo ser víctima por parte de su pareja.

En cuanto al buling de tipo físico, se observa que $68.1 \%$ de los adolescentes del AMM mencionaron que su agresor es un compañero de su escuela; $18.2 \%$ de los casos mencionó a un desconocido y $13.5 \%$ señaló a un familiar, amigo o conocido. Específicamente por tipo de situación, 
$56.5 \%$ de los adolescentes mencionaron que el agresor que les causó daño físico era compañero de la escuela; $15.2 \%$, que era un familiar, amigo o conocido y $14.5 \%$, que era un integrante de su hogar. Respecto a la situación donde se hace daño o se le esconden sus objetos, $75.9 \%$ de los adolescentes mencionó que el agresor era un compañero de la escuela; $9.4 \%$ a un familiar, amigo o desconocido; y $7.3 \%$ a un desconocido. $64.9 \%$ de los adolescentes que son víctimas de robo, mencionaron que el agresor fue un compañero de escuela; $19.2 \%$ que fue un desconocido y $11.4 \%$ a un conocido de vista. En cuanto a la situación de robo con violencia, $31.6 \%$ de los adolescentes mencionó haber sido víctima de esta situación por parte de un familiar, amigo o conocido; $28.2 \%$ mencionó a un compañero de la escuela; $24.4 \%$ a un desconocido; y $15.8 \%$ a un conocido de vista.

El buling de tipo psicológico en los adolescentes del AMM, según 61.3\% de los adolescentes víctimas es causado por un compañero de la escuela; $18.3 \%$ señalaron como agresor a un conocido de vista; $13.1 \%$ a un desconocido; y $7.3 \%$ a un familiar, amigo o conocido. Por tipo específico de situación psicológica, se menciona que la extorsión es ejercida por un compañero de la escuela en todos los casos, mientras que 58.5\% mencionaron que en la situación de amenaza el agresor es un compañero de la escuela; $19.6 \%$ mencionaron como el agresor a un conocido de vista; $14 \%$ a un desconocido; y $7.8 \%$ a un familiar, amigo o conocido.

En cuanto al buling de tipo social, $68.5 \%$ de los adolescentes mencionó que su agresor era un compañero de su escuela; $14.5 \%$ mencionó ser víctima de un familiar, amigo o conocido; $13 \%$ que su agresor era un desconocido; y $9.3 \%$ mencionaron que la persona que se burla, le pone apodos hirientes o lo excluye por su físico, sus gustos o su ropa, era un desconocido.

En cuanto al buling de tipo sexual, se observa que este es el único tipo de buling donde no se menciona como agresor a un compañero de la escuela; $31.5 \%$ de los adolescentes mencionaron como su agresor a un desconocido; $17.3 \%$ a un integrante de su hogar; $16.3 \%$ a un familiar, amigo o conocido; y 9.7\% a un conocido de vista. Por tipo específico de situación, de los adolescentes del AMM que mencionaron que alguien los tocó o intentó tocarlos, 41.7\% mencionó que el agresor fue un desconocido; $21.6 \%$ que fue un familiar, amigo o conocido; y $12.1 \%$ a un integrante de su hogar. Respecto a si alguien lo ha obligado o ha intentado obligarlo a ver o realizar alguna práctica sexual, $29.7 \%$ de los adolescentes víctimas de esta situación, mencionó a un conocido de vista como el agresor; mientras que $24.9 \%$ a un integrante de su hogar.

Finalmente, en cuanto al ciberbuling, 76.4\% de los adolescentes del AMM mencionaron ser víctima de un compañero de la escuela, mientras que $23.6 \%$ señalaron a un desconocido. En este último caso, puede ser que los adolescentes no sepan quién inició la distribución de mensajes de texto, imágenes o videos de su persona, por tanto mencionan que fue alguien desconocido.

En resumen, se observa que los adolescentes de 12 a 16 años del AMM son víctimas de buling físico, psicológico, social y ciberbuling principalmente por parte de un compañero de la 
escuela y en cuanto al buling de tipo sexual, los adolescentes víctimas del mismo mencionaron como su agresor principalmente a un desconocido o a un integrante de su hogar, aunque dependiendo de cuál de las dos situaciones de buling de tipo sexual fue víctima el adolescente, también se mencionó como agresor a un familiar, amigo o conocido y a un conocido de vista.

Se observa que $10.5 \%$ de los adolescentes del AMM que mencionaron haber sido víctimas de tocamiento o intento de tocamiento indeseado de tipo sexual, mencionaron la escuela como el lugar donde sucedió la agresión, sin embargo, como agresor de este hecho no se menciona a los compañeros de escuela y las opciones que aparecen en la encuesta no incluyen a maestros, personal administrativo, intendentes, etc., lo cual podría ser una posibilidad y debería ser revisado con mayor profundidad. En el mismo sentido, destaca el hecho de que $24.9 \%$ de los adolescentes que han sido víctimas de una práctica sexual indeseada, mencionan que el agresor fue un integrante de su hogar, sin embargo, no hay información de dónde sucedió; mientras que en el caso de quienes fueron víctimas de tocamiento o intento de tocamiento, $12.1 \%$ menciona que el agresor fue un integrante de su hogar y $21.6 \%$ que un familiar, amigo o conocido, y $23.2 \%$ de las víctimas señalan que el lugar donde sucedió la agresión fue su propia casa.

\section{Conclusiones}

En el contexto de violencia que se vive en México, donde los jóvenes enfrentan todo tipo de violencia, el buling y el ciberbuling ganan cada vez más espacio entre la problemática de los jóvenes y adolescentes, tal como lo demuestra el que México ocupe el primer lugar en buling entre los países de la OECD.

Por otra parte, la investigación de estos temas en el país también ha estado aumentando en los últimos años, sin embargo, los datos aún son escasos y la mayor parte de la información proviene de estudios de caso, ya sea de corte cualitativo o cuantitativo. Los estudios de caso son muy valiosos y un buen complemento a estudios más generales para una ciudad o estado, pues permiten contar con evidencia para focalizar política pública.

Este estudio busca contribuir a aumentar la información disponible sobre estos temas en el Área Metropolitana de Monterrey a través de un análisis de la incidencia del buling y ciberbuling en los adolescentes de 12 a 16 años. La información obtenida nos permite tener un panorama de cómo se encuentra esta área metropolitana respecto a la media nacional, y conocer cuáles son las situaciones de buling que más se presentan. Asimismo, es representativa para este grupo de edad en el AMM, sin embargo, es información de 2014 pues la ECOPRED es el único instrumento de su tipo en México y no hay una segunda edición del mismo. Aun así, consideramos que esta información es valiosa y nos puede permitir establecer las bases para una política pública encaminada a erradicar el buling en el AMM.

Se obtuvo información de nueve situaciones que se clasificaron según tipo de buling (físico, psicológico, social y sexual) y una situación considerada ciberbuling; se presentó informa- 
ción de la incidencia de cada situación de buling y sus tipos, en general, por género, por lugar donde se presentó y por quién fue el agresor.

El primer resultado que podemos resaltar es que para todas las situaciones de buling la incidencia entre los adolescentes de 12 a 16 años en el AMM es menor que en la media nacional, incluso, en algunos casos la incidencia en la media nacional es el doble que en el AMM, como por ejemplo en ciberbuling. También se observa que mientras en la media nacional las mujeres son las principales víctimas de ciberbuling, en el AMM son los hombres; de aquí la importancia de los estudios como éste, que nos permiten visualizar estas diferencias y responder a la problemática local de forma más certera.

Aunque para muchos investigadores el buling es exclusivo de las escuelas o centros académicos, otros afirman que este tipo de acoso se puede presentar en cualquier ambiente donde niños y adolescentes conviven, incluida la propia casa. Los datos de este estudio nos permiten afirmar que en el AMM, la escuela sí es el principal lugar (aunque no exclusivo) de incidencia de buling entre adolescentes de 12 a 16 años (excepto para el de tipo sexual) y que los compañeros de escuela son señalados como el principal agresor. Así, en el AMM, la escuela se convierte en un lugar estratégico para desarrollar y poner en práctica acciones de prevención.

En el caso del buling de tipo sexual, el principal lugar donde sucede la agresión es la propia casa de la víctima y, dependiendo de la situación, se señala como agresor a un conocido de vista, un desconocido o un integrante de su hogar. De aquí que es necesario desarrollar mecanismos para que los adolescentes puedan estar protegidos en su propia casa y puedan denunciar actos de agresión de tipo sexual de una forma más eficiente y segura. En el mismo sentido, se observa que tanto en el AMM como en la media nacional, la incidencia de mujeres víctimas de buling tipo sexual es casi el doble que la incidencia en hombres, lo que nos indica que este tipo de acoso debe ser considerado en las políticas para combatir y erradicar la violencia contra las mujeres.

Finalmente, podemos mencionar que por género, en el AMM una mayor proporción de mujeres son víctimas de buling tipo físico y sexual, mientras una mayor proporción de hombres son víctimas de ciberbuling. Por otra parte, el buling de tipo psicológico y el social tienen la misma incidencia en hombres y mujeres. De nueva cuenta, estos resultados nos permiten afirmar la importancia de políticas focalizadas al contexto local, pues contrastan con los encontrados en estudios para el país o para otros países, por ejemplo, la UNESCO (2019) menciona que el buling físico es más común en los niños, mientras que el psicológico es más frecuente entre las niñas.

\section{Referencias}

Acosta Ruiz, P. (2014). Teoría epistemológica del bullying: fundamentación teórica. Revista Vinculando. https://vinculando.org/psicologia psicoterapia/teoria-epistemologica-bullying.html 
Aguilera García, M.A.; G. Muñoz Abundez; A. Orozco Martínez (2007), Disciplina, violencia y consumo de sustancias nocivas a la salud en escuelas primarias y secundarias de México. México: INEE.

Anderson, M.; J. Kaufman; T. R. Simon (2001). School-Associated Violent Deaths in the United States. 1994-1999. JAMA, (286), 2695-2702.

Banco Mundial (2012). La violencia juvenil en México. Reporte de la situación, el marco legal y los programas gubernamentales. Washington: Banco Mundial.

Bandura, A. (1973). Aggression: A Social Learning Analysis. Englewood Cliffs: Prentice Hall.

Berkowitz, L. (1969). The Frustration-Aggression Hypothesis Revisted. In Berkowitz, L. (ed.). Roots of Aggression. Nueva York: Atherton Press.

Berkowitz, L; E. Rawling (1963). Effects of Film Violence on Inhibitions against Subsequent Aggression. Journal of Anormal and Social Psychology, (66), 405-412.

Bronfenbrenner, U. (1979). The Ecology of Human Development: Experiments by Nature and Design. Cambridge: Harvard University Press.

Castillo Rocha, C.; M. Pacheco Espejel (2008). Perfil del maltrato (bullying) entre estudiantes de secundaria en la ciudad de Mérida, Yucatán. Revista Mexicana de Investigación Educativa, 13(38), 825-842.

Cobo, P.; R. Tello (2008). Bullying en México. Conducta violenta en niños y adolescentes. México: Cuarzo.

Del Río, J.; X. Bringue; C. Sádaba; D. González (2009). Cyberbullying: un análisis comparativo en estudiantes de Argentina, Brasil, Chile, Colombia, México, Perú y Venezuela. Generació digital: oportunitats i riscos dels públics. La transformació dels usos comunicatius, $V$ Congrés Internacional Comunicació i Realitat. https://dadun.unav.edu/bitstream/10171/17800/1/articulo-cyberbullying.pdf

Dollard, J.; L. Doob; N. Miller; O. Mowrer; R. Sears (1939). Frustration and Aggression. New Haven: Yale University Press.

Domínguez López, F.; M. del C. Manzo Chávez (2011). Las manifestaciones del bullying en adolescentes. Uaricha Revista de Psicología (Nueva época), 8(17), 19-33.

Domínguez Mora, R.; E. Vargas Jiménez; R. Castro Castañeda; R. Medina Centeno; C. Huerta Zúñiga (2019). Ciberacoso como factor asociado al malestar psicológico e ideación suicida en adolescentes escolarizados mexicanos. Acta Universitaria, 29. http://doi.org/10.15174/ au.2019.2295

Fehon, D. (2007). From Victim to Aggressor: The Cycle of Youth Violence. Psychiatric Times, 24(7), 44.

Garaigordobil, M.; J. Mollo-Torrico; E. Larrain (2018). Prevalencia de bullying y cyberbullying en Latinoamérica: una revisión. Revista lberoamericana de Psicología, 11(3), 1-18.

García-Maldonado, G.; G. Martínez-Salazar; A. Saldívar-González; R. Sánchez-Nuncio; G. Martínez-Perales; M. del C. Barrientos-Gómez (2012). Factores de riesgo y consecuencias del 
cyberbullying en un grupo de adolescentes. Asociación con bullying tradicional. Boletín Médico del Hospital Infantil de México, 69(6), 463-474.

García Montañez, M.; Ch. Ascensio Martínez (2015). Bullying y violencia escolar: diferencias, similitudes, actores, consecuencias y origen. Revista Intercontinental de Psicología y Educación, 17(2), 9-38.

Gómez Nashiki, A. (2005). Violencia e institución educativa. Revista Mexicana de Investigación Educativa, 10(26), 693-718.

González Amaya, C.; A. K. Acharya; J. M. Infante Bonfiglio (2018). Bullying y la violencia contra los adolescentes en la familia y su impacto en la agresión en la escuela: Caso de estudio de las preparatorias técnicas en Nuevo León, México. Antropología Experimental, (18), 75-89.

González, P. A.; L. M. Patlán (2009). Bullying: tergiversación de los valores. Revista Alêtheia. Psicología y Psicoanálisis, (28), 15-17.

Griffin, K.; L. Scheier; G. Botvin, T. Díaz; N. Miller (1999). Interpersonal Aggression in Urban Minority Youth: Mediators of Perceived Neighborhood, Peer, and Parental Influences. Journal of Community Psychology, 27(3), 281-298.

Guerrero, R. (2008). ¿Qué sirve en la prevención de la violencia juvenil? Salud Pública de México, 50(1).

Hernández Prados, M. Á.; I. Solano Fernández (2007). Ciberbullying, un problema de acoso escolar. RIED, 10(1), 17-36.

INEE (2009). El aprendizaje en tercero de secundaria en México. Informe sobre los resultados de Excale 09, aplicación 2008. México: INEE.

INEGI (2015). Encuesta de Cohesión Social para la Prevención de la Violencia y la Delincuencia 2014. https://www.inegi.org.mx/rnm/index.php/catalog/148

López-Hernáez, L. (2015). Agresión entre iguales. Teorías sobre su origen y soluciones en los centros educativos. Opción, 31(2), 677-699.

Loredo-Abdalá, A.; A. Perea-Martínez; G. López-Navarrete (2008). Bullying: acoso escolar. La violencia entre iguales. Problemática real en adolescentes. Acta Pediátrica de México, 29(4), 210-214.

Lucio López, L. A. (2009). El cyberbullying en estudiantes del nivel medio superior en México. $X$ Congreso Nacional de Investigación Educativa, Veracruz, México. http://www.comie.org.mx/ congreso/memoriaelectronica/v10/pdf/area tematica 17/ponencias/0901-F.pdf

Mancha Torres, G.; E. Ayala Gaytán (2018). Factores de riesgo asociados a la conducta violenta de los jóvenes en México. Revista Desarrollo y Sociedad, (81), 171-210.

Mendoza González, B.; A. Cervantes Herrera; F. Pedroza Cabrera (2016). Acoso escolar: diferencias en contextos educativos rural y urbano, en alumnado adolescente. Investigación y Ciencia, 24(67), 62-70. 
OECD (2017). PISA 2015 Results (Vol. III): Students' Well-Being. París: PISA, OECD Publishing. https:// www.oecd-ilibrary.org/docserver/9789264273856-en.pdf?expires=1611172154\&id=id\&ac cname $=$ guest \&checksum $=38$ BD0F1E4394DCE384A92038D5D533C8

Olweus, D. (1993). Bullying at School: What We Know and what Can We Do. Oxford: Blackwell. OMS (2002). Informe mundial sobre la violencia y la salud. Washington: OMS.

Ortega, R. (2010). Treinta años de investigación y prevención del bullying y la violencia escolar. En Ortega, R. (ed.). Agresividad injustificada, bullying y violencia escolar. España: Alianza Editorial, 15-32.

Ortega Ruiz, R.; J. Mora-Merchán (1997). Agresividad y violencia. El problema de la victimización entre escolares. Revista de Educación, (313), 7-27, Madrid: INECSE.

Prieto García, M. P. (2005). Violencia escolar y vida cotidiana en la escuela secundaria. Revista Mexicana de Investigación Educativa, 10(27), 1005-1026.

Santoyo Castillo, D.; S. Frías (2014). Acoso escolar en México: actores involucrados y sus características. Revista Latinoamericana de Estudios Educativos, 44(4), 13-41.

Smith, P. K. (2000). Bullying in Schools: Lessons from Two Decades of Research. Aggressive Behavior, (26) 1-9.

Soto, H.; D. Trucco (2015). Inclusión y contextos de violencia. En Trucco, D.; H. Ullmann (eds.). Juventud: realidad y retos para un desarrollo con igualdad. Santiago de Chile: CEPAL. http:// www.cepal.org/es/publicaciones/38978-juventud-realidades-retos-un-desarrollo-igualdad

UNESCO (2019), Behind the Numbers: Ending School Violence and Bullying. París: UNESCO. https:// unesdoc.unesco.org/ark:/48223/pf0000366483

UNODC (2019). Global Study on Homicide 2019. Vienna: United Nations. https://www.unodc.org/ unodc/en/data-and-analysis/global-study-on-homicide.html

Valdés Cuervo, Á.; E. Carlos Martínez; G. Torres Acuña (2012). Diferencias en la situación socioeconómica, clima y ajuste familiar de estudiantes con reporte de bullying y sin ellos. Psicología desde el Caribe, 29(3), 616-631.

Vázquez Valls, R.; A. Villanueva Mercado; A. Rico; M. A. Ramos Herrera (2005). La comunidad de la Preparatoria 2 de la Universidad de Guadalajara. Actitudes de sus miembros respecto de la violencia y no violencia escolar. Revista Mexicana de Investigación Educativa, 10(27), 1047-1070.

Vega Báez, J. A. (2013). La pandemia de bullying en México: políticas sociales urgentes. Revista Trabajo Social, (3131), 73-89.

Vega López, M. G.; G. J. González Pérez (2016). Bullying en la escuela secundaria. Factores que disuaden o refuerzan el comportamiento agresor de los adolescentes. Revista Mexicana de Investigación Educativa, 21(71), 1165-1189. 
Vega López, M. G.; G. J. González Pérez; P. P. Quintero Vega (2013). Ciberacoso: victimización de alumnos en escuelas secundarias públicas de Tlaquepaque, Jalisco, México. Revista de Educación y Desarrollo, 25(2), 13-20. 